\title{
HYDRODYNAMICAL MODEL OF X-RAY EMITTING GAS AROUND ELLIPTICAL GALAXIES
}

\author{
RYO SAITO AND TOSHIKAZU SHIGEYAMA \\ Department of Astronomy, University of Tokyo \\ 7-3-1 Hongo, Bunkyo-ku, Tokyo 113, JAPAN
}

We have performed spherically symmetric, time-dependent hydrodynamical calculations for the $\mathrm{X}$-ray emitting gas around elliptical galaxies, NGC4472, for the purpose to reproduce profiles of temperature and density. Stellar mass loss rate and type Ia supernova rate are assumed to be constant. Thermal conduction is introduced to stabilize against thermal instability due to radiative cooling.

If the gas should evolve in the intracluster medium with $n \sim 10^{-4}$, our model can reproduce the observed density profile (see below). The thermal conduction keeps the gas nearly isothermal for at least $4 \times 10^{9}$ years if the total stellar mass loss rate is less than $1 M_{\odot} /$ year. Thus, we should consider the effect of resonance scattering to create cool component at the center (Shigeyama et al., this volume).

When we assume that part of cooling gas sinks, the rest can reach steady state of central temperature less than $10^{7} \mathrm{~K}$, although amount of cooled gas has not observed by $\mathrm{CO}$ and infrared.
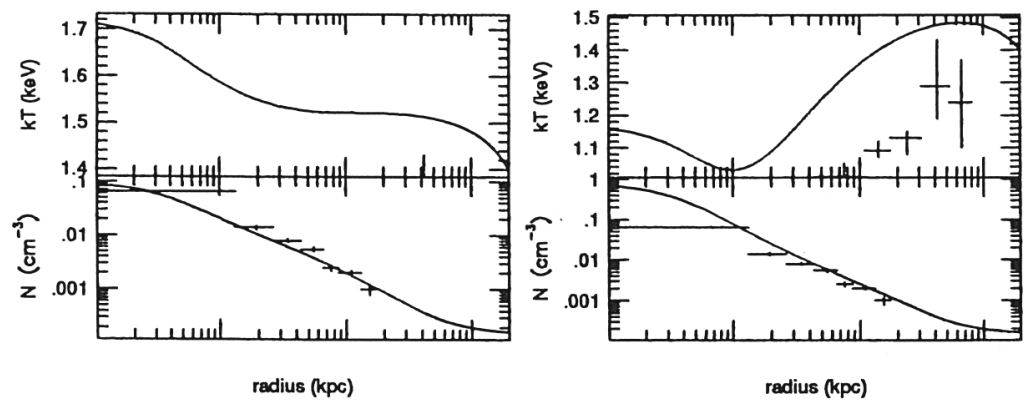

Figure 1. Distributions of temperature and density for the best-fit model at $t=4 \times 10^{9}$ years. The right figure includes sink term. Crosses show ROSAT data (Irvin and Sarazin 1996,ApJ,471,683) 